\title{
Diagnosis of lung disease in acquired immune deficiency syndrome: biopsy or cytology and implications for management
}

\author{
N D FRANCIS, * R D GOLDIN,* S M FORSTER, $\dagger$ H T COOK, ${ }^{*}$ D V COLEMAN, \\ R SHAW, A J PINCHING, $\S$ A W BOYLSTON* \\ From the Departments of ${ }^{*}$ Histopathology, $\uparrow$ Genitourinary Medicine, $\ddagger$ Cytopathology, $\uparrow$ Respiratory Medicine, \\ §Immunology, St Mary's Hospital, London
}

SUMMARY One hundred and twenty consecutive bronchoscopic examinations were carried out on 80 patients with acquired immune deficiency syndrome (AIDS) between January 1982 and December 1986. Ninety one paired biopsy and cytology specimens from 72 of these patients were analysed. There was no significant difference between biopsy and cytology in diagnosing Pneumocystis carinii pneumonia $(0.95>p>0 \cdot 1)$. In 10 cases $P$ carinii pneumonia was diagnosed by biopsy but not cytology and in seven cases by cytology but not biopsy. Nineteen patients had multiple infections or Kaposi's sarcoma. Biopsy was more useful than cytology in the diagnosis of other infections $(n=20)$ and Kaposi's sarcoma $(n=2)$ with positive cytological correlation in only three of the infections.

Biopsy and cytology together have a diagnostic yield of $78.3 \%$. We conclude that all patients presenting with respiratory disease who have, or are in a high risk group for, AIDS should be examined by bronchoscopy at an early stage with both cytology and biopsy.

An increasing number of patients are presenting with respiratory disease as a complication of infection with human immunodeficiency virus (HIV) as a result of an increased susceptibility to bacterial or opportunistic infection. ${ }^{13}$ By January 1987686 cases of acquired immune deficiency syndrome (AIDS) had been reported in the United Kingdom, of which about $50 \%$ presented with $P$ carinii pneumonia (PHLS, unpublished observations). Our own figures showed that respiratory tract biopsies accounted for one third of all biopsies from AIDS patients; of this group $50 \%$ had $P$ carinii pneumonia confirmed by biopsy. ${ }^{4}$ of our first 144 patients fulfilling the Clinical Disease Center (CDC) criteria for AIDS, ${ }^{5} 87$ had severe respiratory complications and 80 of these had bronchoscopy to aid their diagnosis and management.

Up to $80 \%$ of cases of $P$ carinii pneumonia will respond to treatment with co-trimoxazole ${ }^{36}$ but coexistent multiple pathogens are common in AIDS patients ${ }^{7}$ and failure to diagnose these is an important cause of non-response to initial treatment. Further-

Accepted for publication 18 May 1987 more, if diagnosis and appropriate management are delayed until the clinical condition of the patient deteriorates, the response to treatment and prognosis are poor $^{3}$; thus accurate diagnosis before treatment is essential.

A previous short report from this centre, which documented data from some of these patients, indicated that bronchoscopy was valuable in the diagnosis of pneumonia in AIDS, ${ }^{8}$ but more recently others have advocated empirical treatment on clinical grounds with bronchoscopy only when there is deterioration or no response after five days. ${ }^{9}$

In this extended and more detailed series we have reviewed and compared histology and cytology from all the AIDS patients who were bronchscoped between January 1982 and December 1986 to show the importance of these procedures in diagnosis and subsequent management.

\section{Patients and methods}

All fulfilled the revised CDC criteria ${ }^{10}$ for the diagnosis of AIDS either before or as a result of bron- 
choscopy. Patients were considered for bronchoscopy who presented with cough, shortness of breath, chest pain, inability to take full inspiration, or fevers and night sweats. Interstitial shadowing on the chest $x$-ray picture, hypoxia, and a low or deteriorating transfer factor on lung function testing were generally regarded as indications for further investigations. If the clinical picture strongly suggested bacterial infection alone-for example, purulent cough, focal signs on chest examination, lobar consolidation, or a pleural effusion on chest $x$-ray picture-a trial of antibiotics was given after sputum, blood, and pleural fluid had been sent for culture. Severe hypoxia or respiratory distress were regarded as contraindications for bronchoscopy, and platelet concentrations and a clotting screen were always performed before bronchoscopy to exclude thrombocytopenia or a bleeding diathesis, both sometimes seen in HIV infection. ${ }^{11}$ If either was present bronchoscopy with lavage but no biopsy was performed. Informed consent was obtained from the patients and a premedication, usually of papaveretum and atropine, was given intramuscularly one hour beforehand. Occasionally, if the patient was very anxious at the time of the procedure, a further dose of intravenous diazepam was given. The risk of hypoxia during the procedure was minimised by monitoring with oximetry and correcting appropriately.

Biopsy specimens were collected, transported, processed, sectioned and stained as reported previously. ${ }^{4}$

Touch preparations (two to four slides) were made from the biopsy specimen and fixed in $95 \%$ alcohol before staining. Bronchoalveolar lavage fluid and induced sputum were similarly treated by centrifugation for 10 minutes at $1500 \mathrm{rpm}$, the supernatant was decanted, and smears prepared. Two were fixed in alcohol for Papanicolou (PAP) and Grocott staining and one air dried for May-Grünwald-Giemsa stain. Additional smears were made whenever material was available. All unfixed specimens were handled in a class 3 containment cabinet.

All biopsy and cytology reports were reviewed and only those cases with paired biopsy and cytology specimens from the same bronchscopy were included in the study, although all cases were included in the overall figures. All cases with a discrepancy between biopsy and cytology results were again re-examined but the original report in the patients notes was used in the analysis. Statistical analysis was performed using the $\chi^{2}$ test.

Sensitivity was estimated by two different methods to obtain a range. It was assumed that: (i) biopsy and cytology together missed no cases of $P$ carinii pneumonia and from this sensitivities of each method were calculated; (ii) patients with typical clinical features and $x$-ray appearances who responded to "high dose" co-trimoxazole had $P$ carinii pneumonia. From this the sensitivity of each method and combined methods was calculated.

\section{Results}

During the study 120 consecutive bronchoscopies were performed on 80 AIDS patients. From these totals there were 91 paired biopsy and cytology specimens from 72 patients which were included in this study. The 29 unpaired samples comprised 28 biopsy specimens and one broncheolar lavage fluid. The broncheolar lavage fluid was positive for $P$ carinii; 10 biopsy specimens were positive for $P$ carinii, 15 were negative, and three were positive for other infectious organisms.

Table 1 shows how the presence of $P$ carinii was detected by each method. There were eight paired samples that were negative for $\boldsymbol{P}$ carinii pneumonia but in which another infection was diagnosed that were not included in this total. Statistical analysis showed no significant difference betweeen biopsy and cytology in the diagnosis of $P$ carinii pneumonia $(0.95$ $>p>0.1, \chi_{2}=0.5,3 \mathrm{df}$ ). The other main ponts were the high concordance between the results and the fact that $35(43 \%)$ of the paired samples were negative for $P$ carinii. Seventeen of these showed other infections or Kaposi's sarcoma and $18(21 \cdot 7 \%)$ were completely negative. The overall diagnostic yield was therefore $78 \cdot 3 \%$.

Review of the seven false negative biopsy results showed that none contained organisms missed on initial examination, yet in five the positive cytology specimen was a touch preparation. Review of the 10 false negative cytology samples showed two which did contain $P$ carinii (both broncheolar lavage fluid). In three other cases there were scanty organisms in the paired biopsy specimen. In six of the cases the discrepancy was again between biopsy and touch preparations.

Within the group with negative concordance five paired specimens came from four patients, who, on clinical grounds, were strongly thought to have $P$ carinii pneumonia and who responded to "high dose" co-trimoxazole.

Table 2 shows the sensitivity and false negative

Table 1 Paired biopsy and cytology for diagnosing $P$ carinii pneumonia

\begin{tabular}{lllll}
\hline & \multicolumn{2}{l}{ Cytology } & & \\
\cline { 2 - 3 } Biopsy & Positive & & Negative & Totals \\
\hline Positive & 31 & 10 & 41 \\
Negative & 7 & 35 & 42 \\
Totals & 38 & 45 & 83 \\
\hline
\end{tabular}

$\chi^{2}=0.5(\mathrm{df}=3) 0.95>p>0.1$ 
Table 2 Sensitivity of biopsy and cytology in diagnosing P carinii pneumonia (Figures in parentheses indicate percentages)

\begin{tabular}{llclcr}
\hline & \multicolumn{2}{l}{ Laboratory criteria } & & \multicolumn{2}{l}{ Clinical criteria } \\
\cline { 2 - 4 } \cline { 5 - 5 } & Sensitivity & False negative & & Sensitivity & False negative \\
\hline Biopsy & $41 / 48(85 \cdot 4)$ & $7 / 48(14 \cdot 5)$ & $41 / 53(77 \cdot 3)$ & $12 / 53(22 \cdot 7)$ \\
Cytology & $38 / 48(79 \cdot 2)$ & $10 / 48(20 \cdot 8)$ & $38 / 53(71 \cdot 6)$ & $15 / 53(28 \cdot 3)$ \\
Biopsy or cytology & $48 / 48(100)$ & $(0)$ & $48 / 53(90 \cdot 5)$ & $5 / 53(9 \cdot 4)$ \\
\hline
\end{tabular}

rates for each diagnostic method separately and combined, giving a theoretical false negative rate of $0-9 \cdot 4 \%$.

Other diagnoses were usually made on biopsy but a few were made on cytology (table 3). Histoplasma capsulatum, herpes simplex, and giant epithelioid cells (suggesting granulomatous inflammation) were however, detected by cytology. In three cases Candida albicans was detected by cytology but not by biopsy; this may have been due to oral contamination as all three cases were known to have oral candidiasis and none had clinical signs of candidal pneumonia.

Table 4 details the types of cytology specimens from all the cases in which $P$ carinii pneumonia was diagnosed either by cytology or biopsy. Touch preparations and broncheolar lavage fluid were the commonest specimens. None of the specimen types was always positive when $P$ carinii pneumonia was diagnosed, and table 5 shows which cytology specimen types were negative at the same time as another one was positive.

Although this study assessed the biopsy and cytological aspects of the bronchoscopic samples, microbiological investigations were always performed and these resulted in the diagnosis of eight cases of cytomegalovirus, one of adenovirus, and one of herpes simplex. Ten mycobacterial isolates were cultured; nine pyogenic bacteria were isolated; and there were two cases of Cryptococcus sp infection.

Our complication rate with bronchoscopy and biopsy was low. One patient had a haemorrhage of $>100 \mathrm{ml}$, seven developed pneumothorax but only three required chest drains, and 5-10\% had small haemorrhages.

\section{Discussion}

Our results show that biopsy and cytology are complementary diagnostic methods for the diagnosis of $P$ carinii pneumonia and that biopsy is particularly important in the diagnosis of other infections as well as of Kaposi's sarcoma. They also emphasise the incidence of multiple and other infections in AIDS patients; 19 of $80(24 \%)$. Kaposi's sarcoma was commoner in the respiratory tract than this study suggests, because many of the patients were not biopsied in view of the risk of haemorrhage.
Other reports showing the high diagnostic yield and sensitivity of cytological methods alone have concentrated on the diagnosis of $P$ carinii pneumonia ${ }^{12-14}$ and have not highlighted the contribution of biopsy in the diagnosis of other conditions. Our study firmly shows the need for histological examination in addition to cytology.

The sensitivity of each method in detecting $P$ carinii pneumonia ultimately depends on the criteria for diagnosis. We calculated sensitivity using both clinical and laboratory criteria. The former method will underestimate because even the typical presentation of $P$ carinii pneumonia may be mimicked by bacterial and other infections, ${ }^{7}$ which may also be responsive to co-trimoxazole. Using laboratory diagnosis the sensitivity is, by definition, $100 \%$ and is almost certain to be an overestimate. Therefore the range of sensitivity in this study was between 90 and $100 \%$ for biopsy and cytology combined.

Young et al ${ }^{12}$ recently reported a series of immunocompromised patients (none with AIDS) in whom broncheolar lavage fluid was a reliable method for diagnosing $P$ carinii pneumonia as well as detecting two cases of cytomegalovirus and three of Candida. Although this avoids the risk of transbronchial biopsy and has a high diagnostic yield, ${ }^{1314}$ it is unlikely to diagnose other infections-most notably, mycobacterial and bacterial pneumonias, and Kaposi's sarcoma. These conditions can produce a clinical picture indistinguishable from $P$ carinii pneu-

Table 3 Other diagnoses made by biopsy and cytology in 19 patients

\begin{tabular}{lll}
\hline Diagnosis & Biopsy & Cytology \\
\hline Pneumonia/bronchitis & 6 & \\
Acid fast bacilli (AFB) & $5(1)$ & $1^{*}$ \\
Cytomegalovirus (CMV) & $4(2)$ & 1 \\
Herpes simplex & 1 & 1 \\
$\begin{array}{l}\text { Cryptococcus neoformans } \\
\text { Histoplasma capsulatum }\end{array}$ & $1(1)$ & 1 \\
$\begin{array}{l}\text { Candida albicans } \\
\text { Other fungus (unidentified) }\end{array}$ & 1 & 3 \\
Malaria (falciparum) & 1 & \\
Kaposi's sarcoma & 1 & \\
Totals & $2(1)$ & 6 \\
\hline
\end{tabular}

*No AFB but giant cells consistent with granulomatous

inflammation.

Figures in parentheses indicate cases of $\boldsymbol{P}$ carinii pneumonia. 
Table 4 Types of cytology specimens in all patients with tissue diagnosis of $P$ carinii pneumonia

\begin{tabular}{lccc}
\hline $\begin{array}{l}\text { Cytology specimen } \\
\text { Touch preparation }\end{array}$ & $\begin{array}{l}\text { Cytology positive biopsy negative } \\
(n=31)\end{array}$ & $\begin{array}{l}\text { Cytology positive biopsy negative } \\
(n=7)\end{array}$ & $\begin{array}{l}\text { Cytology negative biopsy positive } \\
(n=10)\end{array}$ \\
\hline Bronchoalveolar lavage & 28 & 5 & 6 \\
Induced sputum & 23 & 5 & 8 \\
Totals & 2 & 10 & 0 \\
\hline
\end{tabular}

*One of these cases was subsequently found to have $P$ carinii on review of cytology.

monia, and may coexist with it, but they will require different treatment. Interestingly, cells containing typical cytomegalovirus type inclusions were not found in any of our cytology material even after detailed review. An additional point is that the established risks and complications of transbronchial biopsy are low, ${ }^{15}$ and the improved yield and sensitivity of biopsy and cytology combined from this series outweigh the small risks.

Analysis of the different types of cytology specimens shows that touch preparations and broncheolar lavage fluid are the commonest and that neither is more effective at diagnosing $P$ carinii pneumonia. All specimen types had some false negative results, indicating that even when cytology alone is being relied on more than one type of specimen should be taken.

Surprisingly, of 16 false negative cases, 11 showed a discrepancy between biopsy and touch preparations. This is difficult to explain but may be due to the fact that after touch preparations the biopsy becomes too distorted or crushed to be interpretable or gets "lost" in the teeth of the forceps: or, alternatively, as multiple biopsy specimens are often taken they will not all have touch preparations made from them, and those that have may be too small to be recovered from the pot of formalin.

Many patients will have been given co-trimoxazole at presentation at a time when normal cytology or histology services were not available, up to 60 hours before bronchoscopy. There is evidence that treatment for this length of time and longer does not eradicate the organisms and therefore is unlikely to reduce the diagnostic yield. ${ }^{3}$ Some patients who are too ill to be investigated or have a contraindication to bronchoscopy are diagnosed clinically and treated accordingly.

$$
\text { We and others }{ }^{716-18} \text { do not agree with the }
$$

Table 5 Specimen type and diagnosis of $P$ carinii pneumonia

\begin{tabular}{lll}
\hline Specimen type* & Positive & Negative \\
\hline Touch preparation & 32 & 3 \\
Bronchoalveolar lavage & 28 & 7 \\
Induced sputum & 2 & 3 \\
\hline
\end{tabular}

*When another cytology specimen type from the same bronchoscopy was positive. approach recently suggested by Pozniak et al ${ }^{9}$ for several reasons. Once patients deteriorate, approach respiratory failure, or do not respond to treatment their prognosis is poor. ${ }^{3}$ Failure of response is much more likely to be due to undiagnosed concomitant infection or the poor immune state of the host than to organism resistance. This may be avoided by early and accurate diagnosis with appropriate treatment.

Our results show that nearly $24 \%$ of AIDS patients have other infections, with or without $P$ carinii pneumonia, which emphasises that these patients may receive inappropriate treatment with its associated side effects. ${ }^{6}$ There is also a group of patients (nearly $22 \%$ ) with symptoms and signs warranting bronchoscopy, in whom biopsy and cytology are negative, and most of these would receive five days of unnecessary treatment under the Pozniak strategy.

As has been pointed out ${ }^{18}$ the diagnosis of AIDS is established by $\boldsymbol{P}$ carinii pneumonia and it is therefore important to have positive identification of the organism, especially when it is the only diagnostic criterion in a particular patient. The firm diagnosis of $P$ carinii pneumonia on clinical grounds alone is therefore not justified in view of the profound prognostic, social, psychological and potential medicolegal implications, as well as the question of ventilation.

We consider that early and accurate diagnosis by bronchoscopy with biopsy and cytology are indicated in the management of these patients and this is particularly important in conditions that may mimic $\boldsymbol{P}$ carinii pneumonia. In an environment where AIDS and HIV infection are increasing ${ }^{1920}$ it is important that financial and workload considerations should not interfere with, or compromise, the accurate diagnosis and subsequent optimal treatment of these patients.

\section{References}

1 Rosen MJ, Wy Tow T, Tierstein AS, Chuang MT, Marchevsky A, Bottone EJ. Diagnosis of pulmonary complications of the acquired immune deficiency syndrome. Thorax 1985;40:571-5.

2 Golden JA. Pulmonary disease in the acquired immunodeficiency syndrome. Ann Intern Med 1986;104:286.

3 Murray JF, Felton CP, Garay SM, et al. Pulmonary complications of the acquired immunodeficiency syndrome. $N$ Engl $J$ Med 1984;310:1682-8.

4 Boylston AW, Cook HT, Francis ND, Goldin RD. Biopsy pathology of AIDS. J Clin Pathol 1987;40:1-8.

5 Centres for Disease Control. Update on acquired immunodeficiency syndrome. $M M W R$ 1982;31:507-8. 
6 Young LS. Trimethoprim-sulphamethoxazole in the treatment of adults with pneumonia due to Pneumocystis carinii. Rev Infect Dis 1982;4:608-13.

7 Stover DE, White DA, Romano PA, Gellene RA, Robeson WA. Spectrum of pulmonary diseases associated with the acquired immune deficiency syndrome. Am J Med 1985;78:429-37.

8 Warren JB, Shaw RJ, Weber JN, Holt DA, Keal EE, Pinching AJ. Role of fibreoptic bronchoscopy in management of pneumonia in acquired immune deficiency syndrome. $\mathrm{Br}$ Med $\mathrm{J}$ 1985;291:1012-13.

9 Pozniak AL, Tung KT, Swinburn CR, Tovey S, Semple SJG, McI Johnson N. Clinical and bronchoscopic diagnosis of suspected pneumonia related to AIDS. Br Med J 1986;293:797-9.

10 Anonymous. Revision of the case definition of AIDS for national reporting. [Editorial]. $M M W R$ 1985;34:373-5.

11 Walsh C, Kigel R, Lenette K, Karpatkin S, et al. Thrombocytopenia in homosexual patients. Ann Intern Med 1985; 103:542-5.

12 Young JA, Stone JW, McGonigle RJS, Adu D, Michael J. Diagnosing Pneumocystis carinii pneumonia by cytological examination of bronchoalveolar lavage fluid: a report of 15 cases. $J$ Clin Pathol 1986;39:945-9.

13 Ornstein M, Webber CA, Cash M, Henrich AE. Value of bronchoalveolar lavage in the diagnosis of pulmonary infection in acquired immune deficiency syndrome. Thorax 1986;41:345-9.
14 Stover DE, White DA, Romano PA, Gellene RA. Diagnosis of pulmonary disease in acquired immune deficiency syndrome (AIDS) role of bronchoscopy and bronchoalveolar lavage. $\mathrm{Am}$ Rev Respir Dis 1984;130:659-62.

15 Feldman N. An assessment of transbronchial lung biopsy. $N$ Engl J Med 1975;293:299-300.

16 Hopkin JM, Young JA, Turney JH, Adu D, Michael J. Rapid diagnosis of obscure pneumonia in immunosuppressed renal patients by cytology of alveolar lavage fluid. Lancet 1983;ii:299-301.

17 McDonnell T. Clinical and bronchoscopic diagnosis of suspected pneumonia related to AIDS. Br Med J 1986;293:1502-3.

18 Shaw RJ, Pinching AJ, Keal EE. Clinical and bronchoscopic diagnosis of suspected pneumonia related to AIDS. Br Med J 1986;293:1502.

19 Acheson ED. AIDS: a challenge for public health. Lancet 1986;ii:662-6.

20 McEvoy M, Tillett HE. Some problems in the prediction of future numbers of cases of the aquired immunodeficiency syndrome in the UK. Lancet 1985;ii:541-2.

Requests for reprints to: Dr ND Francis, Department of Histopathology, St Mary's Hospital, Praed Street, London W2 INY, England. 